\title{
O uso de medidas autorrelatadas é válido para a avaliação do estado nutricional da população em geral?
}

\section{Is the use of self-reported measures valid for the evaluation of the nutritional status of the general population?}

Lara Onofre Ferriani ${ }^{1}$

Maria Carmen Viana ${ }^{1}$

${ }^{1}$ Universidade Federal do Espírito Santo, Vitória/ES, Brasil.

\begin{abstract}
A utilização de medidas antropométricas autorrelatadas, como peso e altura, tem sido uma prática comum para a avaliação das condições de saúde e nutrição da população, especialmente em estudos que envolvem grandes amostras. Esse método é indicado uma vez que simplifica a coleta de dados e reduz o custo envolvido na obtenção dessas informações, quando comparado à aferição direta, que demanda equipamentos e pessoal treinado. Destaca-se ainda a importância do monitoramento de tais medidas, já que a prevalência de sobrepeso e obesidade vem crescendo em todo o mundo, e o estado nutricional inadequado está associado a uma série de doenças cardiovasculares e metabólicas ${ }^{1,2}$.

Observa-se, no entanto, que as medidas autorrelatadas são mais imprecisas, quando comparadas às aferidas, já que a precisão do relato é influenciada por diversos fatores como a idade, o sexo, a renda, a escolaridade e o estado nutricional ${ }^{3-5}$. Além disso, tem-se uma tendência à subestimativa do peso corporal e à superestimativa da altura ${ }^{6,7}$, que influenciam a subsequente classificação do estado nutricional, resultando em uma subestimativa da prevalência do excesso de peso populacional.

No Brasil, o programa de Vigilância dos Fatores de Risco e Proteção para Doenças Crônicas por Inquérito Telefônico (Vigitel) ${ }^{8}$ tem empregado informações autorrelatadas ao monitorar a frequência e a distribuição dos principais determinantes das doenças crônicas não transmissíveis. Em sua última avaliação, o Vigitel identificou que 52,5\% dos brasileiros estão acima do peso, sendo que destes, 17,9\% apresentam obesidade. Considerando que as medidas autorrelatadas resultam em estimativas da prevalência de sobrepeso e obesidade significativamente díspares, possivelmente esses percentuais estão subestimados.
\end{abstract}


Assim, faz-se necessário considerar características específicas e individuais que influenciam diretamente o autorrelato, para a aplicação de índices de correção das medidas autorrelatadas e de ajuste, pois a partir delas é avaliado o risco populacional de morbidade, incapacitação e mortalidade prematura por doenças cardiovasculares e metabólicas, decorrentes da inadequação do estado nutricional.

\section{Referências}

1. Organização Mundial da Saúde. Global health risks: mortality and burden of disease attributable to selected major risks [Internet]. Genebra: OMS; 2009. p. 646. Disponível em: URL: < http://www.who.int/healthinfo/global_burden_disease/ GlobalHealthRisks_report_full.pdf>.

2. Obesity: preventing and managing the global epidemic - report of a WHO consultation on obesity. World Health Organ Tech Rep Ser [Internet]. 2000; 894:i-xii, 1-253. Disponível em: URL: <http://www.ncbi.nlm.nih.gov/pubmed/11234459>.

3. Peixoto MRG, Benício MHD, Jardim PCBV. Validade do peso e da altura auto-referidos : o estudo de Goiânia. Rev Saúde Pública. 2006; 40(6):1065-72.

4. Silveira EA, Araújo CL, Gigante DP, Barros AJD, Lima MS. Validação do peso e altura referidos para o diagnóstico do estado nutricional em uma população de adultos no Sul do Brasil. Cad Saúde Pública. 2005; 21(1):235-45.

5. Del Duca GF, González-Chica DA, Santos JV, Knuth AG, Camargo MBJ, Araújo CL. Peso e altura autorreferidos para determinação do estado nutricional de adultos e idosos: validade e implicações em análises de dados. Cad Saúde Publica. 2012; 28(1):75-85.

6. Castro V, Moraes SA, Freitas ICM. Concordância de medidas antropométricas em estudo epidemiológico de base populacional. Rev Bras Epidemiol. 2010; 13(1):58-68.

7. Fonseca MJM, Faerstein E, Chor D, Lopes CS. Validade de peso e estatura informados e índice de massa corporal: estudo pró-saúde. Rev Saúde Pública. 2004; 38(3):392-8.

8. Brasil. Ministério da Saúde. Vigitel Brasil 2014: vigilância de fatores de risco e proteção para doenças crônicas por inquérito telefônico. Brasília: Ministério da Saúde; 2014. 